DOI: $10.1515 /$ awutp -2015-0210

\title{
THE EFFECT OF PARTICLE CONCENTRATION ON THE HEATING RATE OF FERROFLUIDS FOR MAGNETIC HYPERTHERMIA
}

\author{
I. Malaescu $^{1 *}$, C. N. Marin ${ }^{1}$, M. Bunoiu ${ }^{1}$, P. C. Fannin ${ }^{2}$, N. Stefu ${ }^{1}$, L. Iordaconiu ${ }^{1}$ \\ ${ }^{1}$ West University of Timisoara, Faculty of Physics, B-dul V. Parvan, no. 4, 1900 Timisoara, Romania \\ ${ }^{2}$ Department of Electronic and Electrical Engineering, Trinity College, Dublin 2, Ireland
}

Article Info

Received: 19.10 .2015

Accepted: 21.11.2015

Keywords: Ferrofluids, Complex magnetic susceptibility, Heating rate, hyperthermia applications.

\begin{abstract}
The complex magnetic susceptibility $\chi(f)=\chi^{\prime}(f)-i \chi^{\prime \prime}(f)$, of a ferrofluid sample with magnetite particles dispersed in kerosene and stabilized with oleic acid, over the range $0.1 \mathrm{GHz}$ to $6 \mathrm{GHz}$, was determined. The initial sample has been successively diluted with kerosene (with a dilution rate of $2 / 3$ ), thus obtaining further three samples. Using the complex magnetic susceptibility measurements of each sample, the frequency field and particle concentration dependencies of the heating rate of the ferrofluid samples, were analyzed. The results show the possibility of using the heating rate of ferrofluid samples with different particle concentrations, in hyperthermia applications.
\end{abstract}

\section{Introduction}

Ferrofluids are stable colloidal systems consisting of single-domain magnetic particles dispersed in a carrier liquid [1]. In order to prevent agglomeration, the magnetic particles are coated with a surfactant. These systems have been the subjects of many research studies, both for applications and for fundamental research because their properties can be controlled during the manufacturing process [2]. The properties of ferrofluids or the properties of the magnetic particles within of ferrofluids can be investigated by the dilution series method used in different type of measurements [3 - 5]. For the characterization of a ferrofluid sample in static magnetic field, the saturation magnetization $\left(M_{\infty}\right)$, the magnetic diameter $\left(d_{m}\right)$ of particles and the particles concentration $(n)$ from the ferrofluid sample must be experimentally determined by means of magnetization curve measurement in a similar manner as is described in [6]. Generally, the dynamic properties of ferrofluids (relaxation processes and ferromagnetic resonance) can be evaluated using the complex magnetic susceptibility measurements into a wide frequency range [7-10]. Due to the interaction of the variable 
magnetic field with the ferrofluid, energy absorption occurs [11]. Following, the ferrofluid is heated and the heating rate is given by the practical equation proposed in [12]:

$$
\frac{\Delta T}{\Delta t}=\frac{p}{(1-\varphi) \rho_{L} c_{L}+\varphi \rho_{S} c_{S}}
$$

In Eq. (1), $\Delta T$ is the increase in temperature; $\Delta t$ is the heating time interval; $c_{L}$ and $\rho_{L}$ are the specific heat and the density of the basic-liquid of the ferrofluid; $c_{S}$ and $\rho_{S}$ are the specific heat and the density of the solid fraction of the ferrofluid; $\varphi$ is the volume fraction of the ferrofluid and $p$ is the specific power dissipation in ferrofluid [10-12], being computed with the following equation:

$$
p=\pi \mu_{0} \chi^{\prime \prime} f H_{0}^{2}
$$

In Eq. (2), $H_{0}$ is the amplitude of the magnetic field; $f$ is the frequency of the applied field; $\mu_{0}$ is the free-space permeability; and $\chi^{\prime \prime}$ is the imaginary component of the complex magnetic susceptibility of the ferrofluid.

In this paper, using the complex magnetic susceptibility measurements in the frequency range between $(0.1-6) \mathrm{GHz}$, we have investigated the frequency and particle concentration dependences of the heating rate of the ferrofluid samples.

\section{Samples and experimental}

The initial sample was a ferrofluid denoted S1, with magnetite particles dispersed in kerosene and stabilized with oleic acid. Starting from the initial ferrofluid S1, three samples denoted S2, S3 and S4 were obtained by successive dilution with kerosene at a dilution ratio $2 / 3$.

The static magnetization measurements were performed using an inductive method [13]. From the static measurements, a Langevin type dependency [1] of the magnetization $M$ on the magnetic field $H$ was observed for the investigated samples. Based on the $M(H)$ dependencies and using the Chantrell method [14] for the magneto-granulometric analysis, the saturation magnetization of the ferrofluid $\left(M_{\infty}\right)$, the concentration of particles $(n)$ and the average magnetic diameter of particle $\left(d_{m}\right)$ were determined. It is known that $M_{\infty}$ does not depend on the interactions between particles or possible particle agglomerations within the ferrofluid sample [1]. Therefore, the values of the saturation magnetization of the ferrofluid samples are the values which were determined from the static measurements and magneto-granulometric 
analysis. The following results were obtained: $M_{\infty}(S 1)=9.11 \mathrm{kA} / \mathrm{m}, n(S 1)=2.52 \cdot 10^{22} \mathrm{~m}^{-3}$; $M_{\infty}(S 2)=6.05 \mathrm{kA} / \mathrm{m}, n(S 2)=1.70 \cdot 10^{22} \mathrm{~m}^{-3} ; M_{\infty}(S 3)=4.10 \mathrm{kA} / \mathrm{m}, n(S 3)=1.15 \cdot 10^{22} \mathrm{~m}^{-3}$; $M_{\infty}(S 4)=2.74 \mathrm{kA} / \mathrm{m}, n(S 4)=0.77 \cdot 10^{22} \mathrm{~m}^{-3}$.

The saturation magnetization of a ferrofluid, $M_{\infty}$, is correlated with the spontaneous magnetization of the bulk material from which the particles originate $M_{S}$, by the relation:

$$
M_{\infty}=\varphi_{m} M_{S}
$$

where $\varphi_{m}$ represents the magnetic volume fraction of the particles from the ferrofluid which is correlated with the volume fraction, $\varphi$ by the following relation $[1,12]$ :

$$
\frac{\varphi_{m}}{\varphi}=\frac{1}{\left(1+\frac{2 \delta}{d_{m}}\right)^{3}}
$$

where $\delta$ is the thickness of the surfactant layer $(\delta \cong 2 \mathrm{~nm})$ [1].

On the other hand, due to the interactions between the particles or to the possible particle agglomerations from the ferrofluid sample, the average magnetic diameter of particles $d_{m}$, obtained by magneto-granulometric analysis, depends on the particle concentration of the sample. As a result, for all the investigated samples we considered the same magnetic diameter $d_{m}$, which corresponds to the most diluted sample (sample S4), where interactions between particles are practically negligible. From the magneto-granulometric analysis of the sample $\mathrm{S} 4$, the value obtained is $d_{m}=9.8 \mathrm{~nm}$. Using the obtained values for the saturation magnetization $M_{\infty}$ of the ferrofluid samples, equations (3) and (4) and taking into account that $M_{S}$ for magnetite particle is $M_{S}=477.5 \mathrm{kA} / \mathrm{m}$ [1], we have computed the following values of the volume fraction, $\varphi$, of the particles from the ferrofluid samples: $\varphi_{1}=5.32 \%, \varphi_{2}=3.53 \%$, $\varphi_{3}=2.42 \%, \varphi_{4}=1.60 \%$.

The measurements of the complex magnetic susceptibility in the frequency range between $0.1 \mathrm{GHz}$ to $6.0 \mathrm{GHz}$ were made by means of the short-circuited coaxial transmission line technique [15], by using a HP $50 \Omega$ coaxial line incorporating a coaxial cell, in conjunction with a network analyzer (HP 8753C type) [15]. 


\section{Results and discussions}

The frequency dependence of the real $\left(\chi^{\prime}\right)$ and imaginary $\left(\chi^{\prime \prime}\right)$ components of the complex magnetic susceptibility in the frequency range between $0.1 \mathrm{GHz}$ to $6.0 \mathrm{GHz}$ at different particle concentrations $(n)$ in the ferrofluid samples is shown in figure 1.

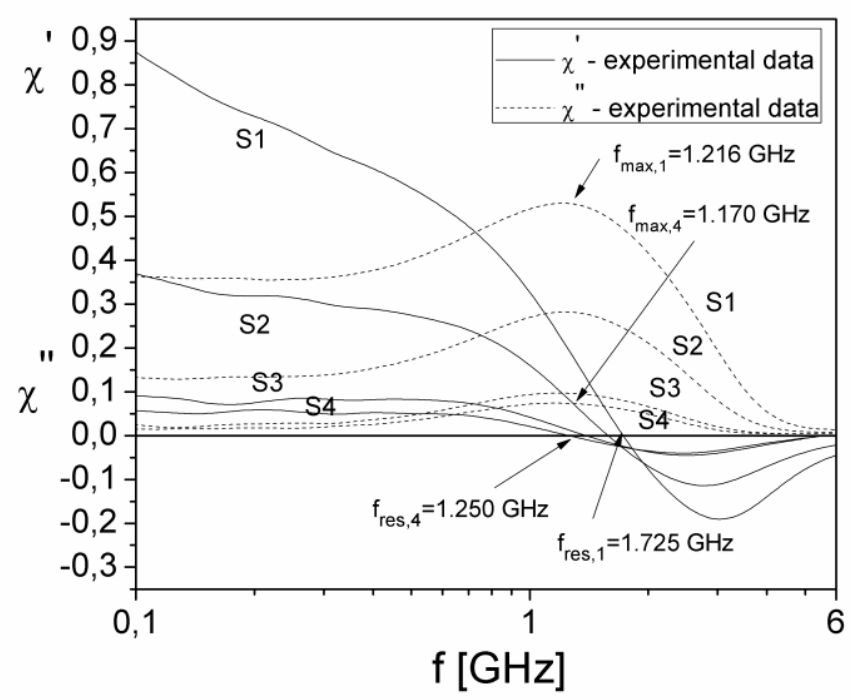

Fig. 1. The frequency and particle concentration dependence of the complex magnetic susceptibility for ferrofluid samples

As can be observed from figure 1 , the imaginary component $\chi^{\prime \prime}$ has a maximum at a frequency $f_{\max }$ for each sample. By decreasing the particle concentration, $f_{\max }$ is moving towards smaller values from $f_{\max , 1}=1.216 \mathrm{GHz}$ to $f_{\max , 4}=1.170 \mathrm{GHz}$. All samples present a ferromagnetic resonance phenomenon, shown by the transition of the real part of the complex magnetic susceptibility $\chi^{\prime}$, from positive values to negative values, at a frequency $f_{\text {res }}$, named the resonance frequency $[9,10]$. By decreasing the particle concentration, $f_{\text {res }}$ is moving towards smaller values, from $f_{\text {res }, 1}=1.725 \mathrm{GHz}$ to $f_{\text {res }, 4}=1.250 \mathrm{GHz}$.

Using the experimental values obtained for the imaginary component $\chi^{\prime \prime}$ from figure 1 and equation (2), we have computed the specific loss power of the ferrofluid samples. For the amplitude $H_{0}$ of the magnetic component of electromagnetic field, we assumed the value $H_{0}=1 \mathrm{~A} / \mathrm{m}$. In figure 2 it is shown the frequency and particle concentration dependence of the specific loss power $(p)$ for the investigated samples. 


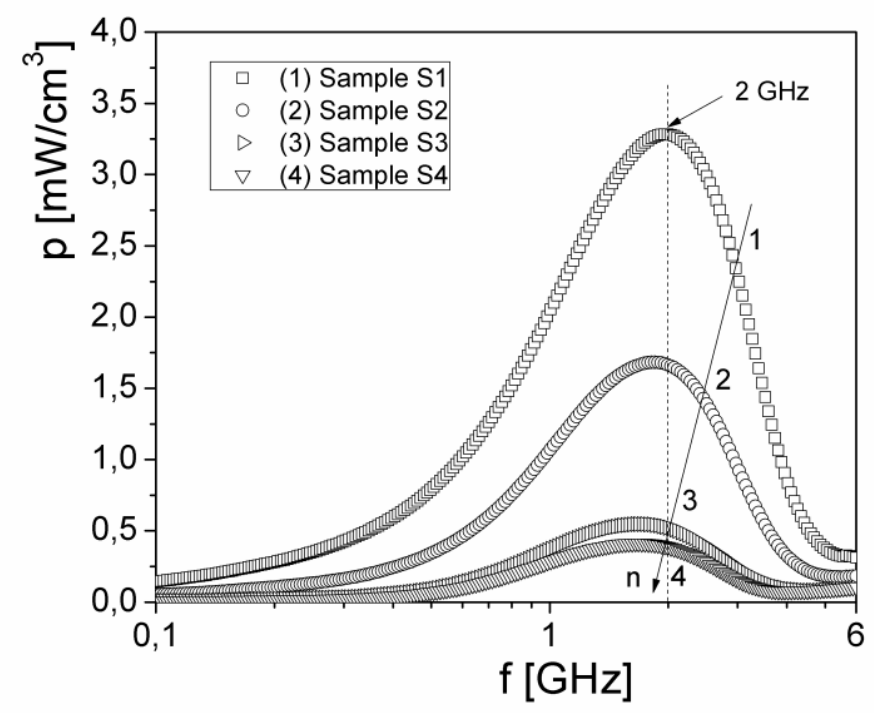

Fig. 2. The frequency and particle concentration dependence of the specific loss power for ferrofluid samples

As can be observed from figure 2, the specific loss power $p$, has a maximum at a frequency $f_{\max }$, for all the particle concentrations from the ferrofluid samples. By decreasing the particle concentration, $f_{\max }$ is moving towards smaller values.

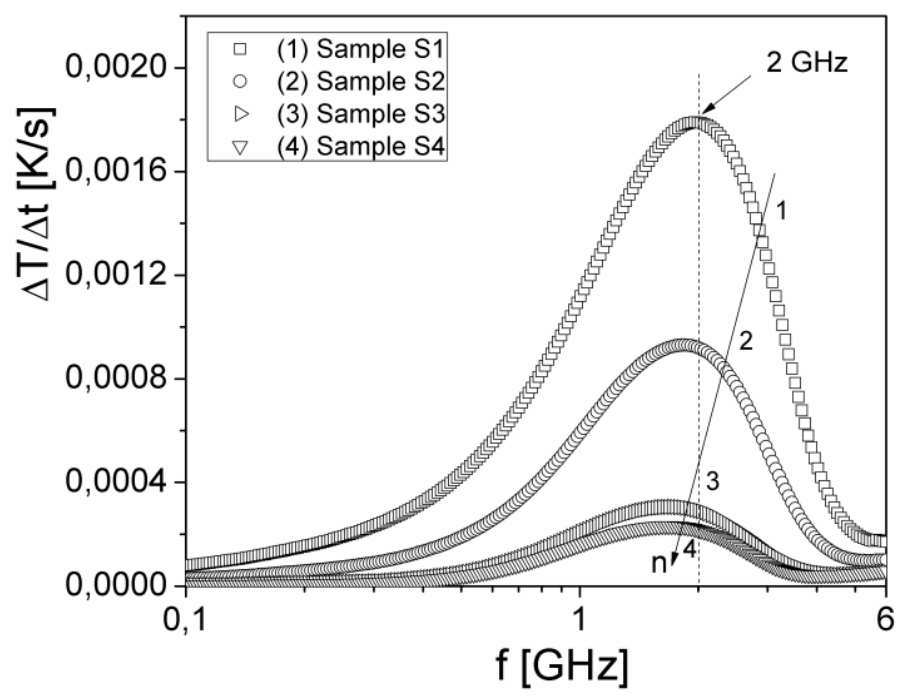

Fig. 3. The frequency and particle concentration dependence of the heating rate of ferrofluid samples

Using the experimental values obtained for the specific loss power (figure 2) and equation (1), we have computed the heating rate $(\Delta T / \Delta t)$ of the ferrofluid samples, taking into account the values computed for the volume fraction of the each sample $(\varphi)$. The 
investigated sample is a ferrofluid with magnetite particles dispersed in kerosene. As a result, in Eq. (1) we have used for the specific heat $c_{L}$ and the density $\rho_{L}$ of the basic-liquid (kerosene) or for the specific heat $c_{S}$ and the density $\rho_{S}$ of the solid fraction (magnetite), the following values: $\rho_{L}=840 \mathrm{Kg} / \mathrm{m}^{3}, \quad c_{L}=2080 \mathrm{~J} / \mathrm{Kg} \cdot \mathrm{K}, \quad \rho_{S}=5200 \mathrm{Kg} / \mathrm{m}^{3}$, $c_{S}=670 \mathrm{~J} / \mathrm{Kg} \cdot \mathrm{K}$.

In figure 3 it is shown the frequency and particle concentration dependence of the heating rate of the ferrofluid samples. From figure 3 it is observed that the heating rate shows a maximum for all samples at frequencies which are found in the range (1-2) GHz, depending on the concentration of the particles. The maximum amplitude of the heating rate increases by increasing the concentration of particles due both of specific power $(p)$ and frequency $f$ of the electromagnetic field (see Eq. 2). This result may provide an opportunity to control the heating rate $(\Delta T / \Delta t)$ through the particle concentration of the ferrofluid with applications in hyperthermia.

Figure 4 shows the time $(t)$ dependence of the increase in temperature $(\Delta T)$ for the investigated ferrofluid samples (having different concentrations) at two frequency values of the field: $f=1 \mathrm{GHz}$ and $f=2 \mathrm{GHz}$.

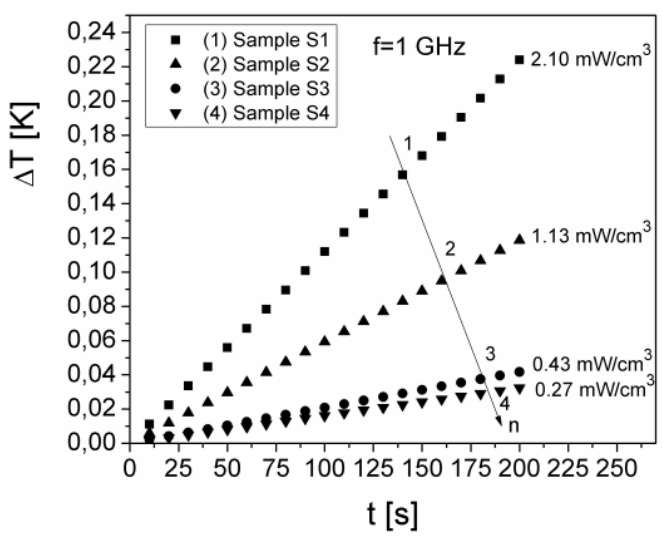

a)

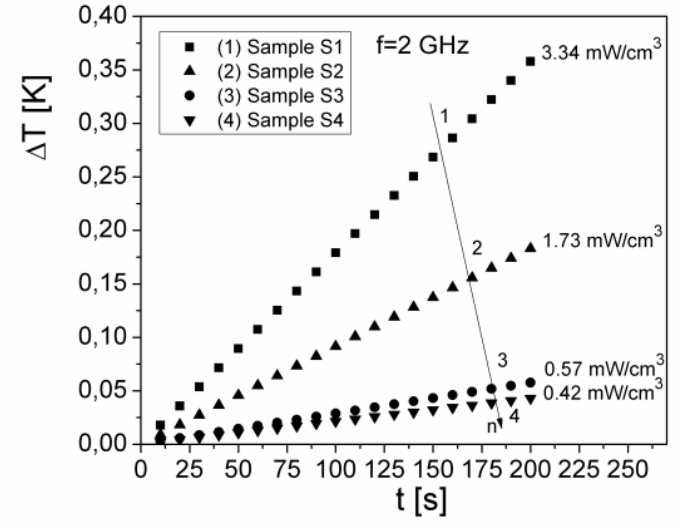

b)

Fig. 4. The time dependence of the heating rate of ferrofluid samples at two constant frequencies: $f=1 \mathrm{GHz}(\mathrm{a}) ; f=2 \mathrm{GHz}$ (b)

As can be observed from figure 4 , the increase in temperature $(\Delta T)$ has a linear dependence on time $(t)$, the greater the slope of the line the higher the particle concentration of ferrofluid. Also, from figure $4 \mathrm{a}$ ) and b), it can be observed that $\Delta T$ depends on the field frequency. Thus, in a microwave field with an amplitude of $1 \mathrm{~A} / \mathrm{m}$, for sample $\mathrm{S} 1$ (with the largest particle concentration) at $f=2 \mathrm{GHz}$ and an application time of the variable 
electromagnetic field of $t=200 \mathrm{~s}$, the increase in temperature $(\Delta T)$ is $0.35 \mathrm{~K}$, while at $f=1$ $\mathrm{GHz}$, for the same sample S1 and the same time duration $(200 \mathrm{~s})$, the increase in temperature is only $0.22 \mathrm{~K}$. This is due to the fact that the absorption of the specific power from the microwave field is greater at higher frequencies and larger concentration of particles (see fig. 4). The results presented in figure 4 show that the same value of the increase in temperature can be achieved using a diluted ferrofluid, only if it is subjected for a longer time to the microwave field. Also, if the amplitude of the magnetic field $H_{0}$ increases 5 times (easily obtained in practice) the specific loss power, computed with eq. (2) increases 25 times. As a result, the heating rate of the ferrofluid increases and therefore $\Delta T$ (see fig. 4) will increase 25 times. Thus, for sample $\mathrm{S} 1$ the increase in temperature $(\Delta T)$ is $8.75 \mathrm{~K}$ (at $f=2 \mathrm{GHz}$ and $t=200$ $s$ ) and $5.5 \mathrm{~K}$ (at $f=1 \mathrm{GHz}$ and $t=200 \mathrm{~s}$ ). These results can be used in magnetic hyperthermia applications.

\section{Conclusions}

In this paper starting with a ferrofluid sample with magnetite particles dispersed in kerosene and stabilized with oleic acid, other three samples were obtained by successive dilution with kerosene (dilution ratio 2/3). For all samples the frequency dependence of the complex magnetic susceptibility $\chi(f)=\chi^{\prime}(f)-i \chi^{\prime \prime}(f)$, over the range $0.1 \mathrm{GHz}$ to $6 \mathrm{GHz}$, was determined.

Based on the complex magnetic susceptibility measurements, the frequency and particle concentrations dependencies of the heating rate of the ferrofluid samples were analyzed. The heating rate presents a maximum for all samples at specific frequencies placed in the range (12) $\mathrm{GHz}$, depending on the concentration of the particles.

The increase in temperature $(\Delta T)$ has a linear dependence on time $(t)$ and it depends on the field frequency and on the particle concentration of ferrofluid. The results show that in a diluted ferrofluid, the same value $\Delta T$ can be obtained as in a more concentrated ferrofluid, only if it is subjected to the microwave field for a longer time.

The obtained results suggest the possibility to control the heating rate $(\Delta T / \Delta t)$ of a ferrofluid in a microwave field by changing the particle concentration of the ferrofluid with applications in magnetic hyperthermia. 


\section{Acknowledgment}

Authors acknowledge the financial support from the research project JINR-Romania protocol no. 4434-4-15/17, order no. 34/23.01.2015 item 50 and 49, Theme JINR 04-4-1121$2015 / 2017$.

\section{References}

[1] R. E. Rosensweig, Ferrohydrodynamics, Cambridge University Press, Cambridge, 1985.

[2] S. Odenbach, S. Thurm, K. Melzner, J. Magn. Magn. Mater., 252 (2002) 421

[3] M.Rasa, D. Bica, A. Philipse, L. Vekas, $9^{\text {th }}$ International Conference on Magnetic Fluids, Bremen, $23^{\text {rd }}-27^{\text {th }}$ July, 2001, Book of Abstacts.

[4] I. Hrianca, I. Malaescu, F. Claici, C. N. Marin, J. Magn. Magn. Mater., 201 (1999) 126.

[5] C. N. Marin, I. Malaescu, A. Ercuta, J. Phys. D: Appl. Phys., 34 (2001) 1466.

[6] R. W. Chantrell, J. Popplewell, S. W. Charles, IEEE Trans. Magn. 14 (5) (1978) 975.

[7] I. Malaescu, C.N. Marin, Physica B: Condensed Matter, 365 (1-4) (2005) 134-140

[8] P. C. Fannin, C .N. Marin, I. Malaescu, N. Stefu, Physica B: Condensed Matter, 388 (1-2) (2007) $87-92$

[9] P.C. Fannin, I. Malaescu, C. N. Marin, J.Magn.Magn.Mater., 289 (2005) 162 - 164

[10] P. C. Fannin, I. Malaescu, C.N. Marin, N. Stefu, Eur. Phys. J. E., 27(2) (2008) 145-1488

[11] R. E. Rosensweig, J. Magn. Magn. Mater., 252 (2002) 370-374

[12] C. N. Marin, I. Malaescu, P. C. Fannin, J. Therm. Anal. Calorim., 119 (2015) 11991203

[13] I. Mihalca, A. Ercuta, C. Ionascu, Sensors and Actuators A: Physical, 106, 1-3, (2003), $61-64$

[14] R. W. Chantrell, J. Popplewell, S. W. Charles, IEEE Trans. Magn., 14, (1978), 975

[15] P. C. Fannin, T. Relihan, S. W. Charles, J. Phys.D: Appl. Phys., 28, 2003 (1995) 